\title{
Nachruf auf Prof. Dr. Dr. h. c. Rolf Lieberwirth
}

\author{
* 1. 12. 1920 Halle an der Saale; $\uparrow$ 5. 4. 2019 Halle an der Saale
}

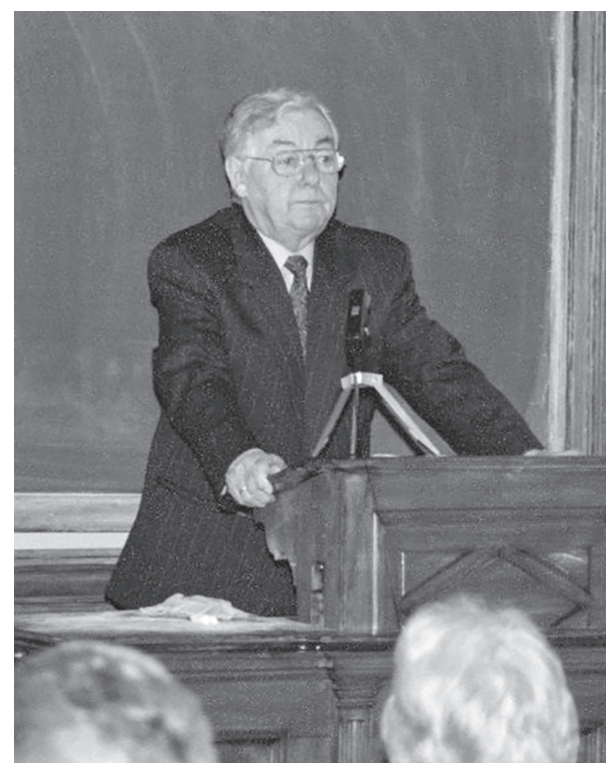

Rolf Lieberwirth bei einem Vortrag 1994.

Foto: Bild- und Filmstelle der Martin-Luther-Universität Halle-Wittenberg / S. Schulze
Die Juristische und Wirtschaftswissenschaftliche Fakultät der Martin-Luther-Universität Halle-Wittenberg trauert um ihr ehemaliges Mitglied Prof. Dr. Dr. h. c. Rolf Lieberwirth. Rolf Lieberwirth war ein herausragender Forscher und Hochschullehrer auf dem Gebiet der Deutschen Rechtsgeschichte. Die von ihm in Halle vertretene Wissenschaftsdisziplin erlangte während der zweiten Hälfte des 20. Jahrhunderts internationales Ansehen. $\mathrm{Zu}$ seinen Forschungsschwerpunkten gehörten insbesondere der berühmte Jurist und Philosoph Christian Thomasius (1655-1728), die Wissenschafts- und Universitätsgeschichte der Aufklärungszeit sowie die Entstehungsund Wirkungsgeschichte des Sachsenspiegels. Hierzu hat er Grundlegendes und Dauerhaftes geleistet. Von den viel beachteten Werken sind die immer noch maßgebliche Thomasius-Bibliographie (1955), die Übersetzung der Thomasius-Dissertationen über

die Hexenprozesse und die Folter sowie essentielle Studien zum Sachsenspiegel und zu seinen Glossen hervorzuheben.

Rolf Lieberwirth wurde am 1. Dezember 1920 in Halle an der Saale geboren. Nach dem Schulbesuch wurde der 19jährige zum Militärdienst einberufen. Er hat den Zweiten Weltkrieg als Soldat von Anfang bis Ende miterlebt. Vor allem hat er ihn überlebt, was angesichts der menschenverachtenden Umstände ein großes Glück war. Eine schwere lebensgefährliche Verwundung konnte dank ärztlicher hoher Kunst geheilt werden. Gleich nach der Wiedereröffnung der hallischen Universität 1946 nahm Rolf Lieberwirth das Studium der Rechtswissenschaft auf. Seit 1948 wirkte an der damaligen Juristischen Fakultät die renommierte Rechtshistorikerin Prof. Dr. Gertrud Schubart-Fikentscher (1896-1985). 
Lieberwirth wurde nach dem Examen ihr Assistent und begann in dieser Position seine wissenschaftliche Laufbahn. Der 300. Geburtstag von Christian Thomasius (1955) stand bevor. So fand Rolf Lieberwirth unter der Ägide seiner verehrten Lehrerin über Christian Thomasius den wissenschaftlichen Zugang zum Recht und zur Ideenwelt der Aufklärungszeit. 1953 folgten die Promotion mit einer Dissertation über die Pfandrechte in der Zeit der Aufklärung sowie 1967 die Habilitation zu grundlegenden Schriften von Christian Thomasius. Seit dieser Zeit galt Rolf Lieberwirth als Thomasiusspezialist und war international hoch anerkannt. Seine Art und Weise, Rechtsgeschichte auf hohem Niveau zu betreiben, zeichnete sich dadurch aus, dass er stets eine philologisch-historische Herangehensweise pflegte, was ihm eine hohe Akzeptanz bei und fruchtbare Kooperationsmöglichkeiten mit anderen geisteswissenschaftlichen Disziplinen eröffnete.

Von 1961 bis zu seiner Emeritierung 1986 lehrte und forschte Rolf Lieberwirth als Professor für Deutsche Rechtsgeschichte und Internationales Privatrecht an der Martin-Luther-Universität Halle-Wittenberg. Hier wirkte er als sehr anerkannter Hochschullehrer und Forscher, dessen Bekanntheitsgrad auch im Westen Deutschlands, der damaligen Bundesrepublik, erheblich war. Er war einer der wenigen Hochschullehrer auf dem Gebiet der Rechtswissenschaft, die dem bürgerlich-humanistischen Bildungsideal die Treue hielten und sich nicht von der herrschenden Ideologie vereinnahmen ließen.

Diese Grundhaltung hat Rolf Lieberwirth, soweit das unter den schwierigen Umständen möglich war, auch seinen Schülern und Studenten vermittelt. Seine wissenschaftliche und charakterliche Akzeptanz in der Bundesrepublik Deutschland sowie im europäischen Ausland (Österreich, Schweiz, Frankreich, Polen, Ungarn, Tschechien, Slowakei) war über alle Zweifel erhaben. Mit dem ehemaligen Rektor der Karlsuniversität zu Prag, Prof. JUDr. Karel Malý DrSc. Dr. h. c., war Lieberwirth eng kollegial und kooperativ verbunden.

Mit der politischen Wende 1989/90 in der DDR erfüllte sich ein Ideal des engagierten Hochschullehrers und Forschers Rolf Lieberwirth, das vor allem den Wiedereinzug der Freiheit von Lehre und Forschung an der Martin-Luther-Universität beinhaltete. Beim Neuaufbau der Juristischen Fakultät an Stelle der 1990/91 abgewickelten „Sektion Staats- und Rechtswissenschaft" erwarb sich Rolf Lieberwirth hervorragende Verdienste. Zunächst wurde er gebeten, mit immerhin über 70 Lebensjahren, wieder Vorlesungen über Deutsche Rechtsgeschichte zu halten. Diese Aufgabe erfüllte er mit Bravour.

Des Weiteren wurde er als fachlich geachteter Professor der Rechtswissenschaft dafür gewonnen, in der Gründungskommission für die 1991 ff. neu aufzubauende Juristische Fakultät mitzuwirken. In diesem Gremium leistete er eine grundlegende Arbeit, die auf die Besetzung der neuen (Gründungs-) Professuren zielte. Dank seines Engagements und seiner fachlichen Kompetenz konnte in kurzer Zeit eine mit anderen deutschen Juristenfakultäten konkurrenzfähige Besetzung erreicht werden. 1993 fand die feierliche Gründungsveranstaltung der neuen Juristischen Fakultät Halle-Wittenberg statt.

Die Mitwirkung von Rolf Lieberwirth in dieser entscheidenden Gründungsphase war auch unter dem Gesichtspunkt der gemeinsamen Gestaltung der neuen freiheitlich-demokratischen Verhältnisse durch Personen aus den ,alten Bundesländern“ und Personen aus den „neuen Bundesländern“ von weit reichender politischer und sozialer Bedeutung. So konnten viele Vorurteile gegenüber den neuen Strukturen und Grundlagen von vornherein abgebaut bzw. minimiert werden. Auch an der Neugründung der Juristischen Fakultät an der Universität Rostock wirkte Lieberwirth als Gründungskommissionsmitglied mit. 
Neben der verantwortungsvollen Tätigkeit als Mitglied der Gründungskommission für die Juristische Fakultät wurde Rolf Lieberwirth 1989 vom Rektor der Martin-Luther-Universität gebeten, die Rehabilitierungskommission der Universität zu leiten. Diese Aufgabe nahm Rolf Lieberwirth ebenfalls mit großem Engagement und menschlichem Einfühlungsvermögen wahr. Die Kommission hatte die Aufgabe, Wissenschaftler, die wegen ihrer politischen Einstellung an ihrem akademischen Werdegang in der DDR benachteiligt worden waren (Nichtzulassung zur Habilitation, Zurücksetzung bei Stellenbesetzung etc.), seitens der Universität zu rehabilitieren. Auch hierbei handelte es sich um eine Aufgabe, von deren Realisierungsqualität das Werden der deutschen Einheit im Bereich der Wissenschaft abhing. In dieser Funktion war Rolf Lieberwirth mehrere Jahre tätig. Dank seiner umsichtigen, gleichwohl engagierten Amtsführung konnten viele Wissenschaftler(innen) ihren akademischen Verdiensten gemäß rehabilitiert werden. Die Wirkung dieser Unternehmung zur Beseitigung bzw. Kompensierung von DDR-Unrecht kann aus der Retrospektive kaum überschätzt werden. Auch daran soll nach knapp 30 Jahren anlässlich des Todes von Rolf Lieberwirth erinnert werden.

Als weiteres Wirkungsfeld von Rolf Lieberwirth muss die Sächsische Akademie der Wissenschaften zu Leipzig, die seit 1945 für die Länder Sachsen, Sachsen-Anhalt und Thüringen als renommierte Gelehrtengesellschaft zuständig ist, aufgerufen werden. Im Jahre 1972 ist er zum Ordentlichen Mitglied der Sächsischen Akademie gewählt worden. Hier entfaltete er in der Philologisch-historischen Klasse, aber auch im Plenum, eine rege Vortrags-, Diskussions-, und Publikationstätigkeit. Seine in den Sitzungsberichten der Akademie publizierten Akademievorträge fanden große Beachtung. Analoges gilt für Lieberwirths Wirken in der der Akademie angeschlossenen Historischen Kommission, welche als einzige Historische Kommission auch in der DDR-Zeit existierte.

In den frühen 1990er Jahren hatte Rolf Lieberwirth als Vizepräsident der Akademie unter anderem die personelle Erneuerung der Gelehrtengesellschaft mit zu leiten. Die damit verbundenen Probleme waren ähnlich jenen, die an den Universitäten zu bewältigen waren. Die personelle Erneuerung war existentiell für den Fortbestand der Akademie. Nur auf diesem Weg konnte sie einen geachteten Platz neben den weiteren sechs Akademien (Berlin-Brandenburgische Akademie, Nordrhein-westfälische Akademie, Akademie der Wissenschaften zu Göttingen, Heidelberger Akademie der Wissenschaften, Akademie der Wissenschaften und Literatur Mainz, Bayerische Akademie der Wissenschaften) finden. Das ist dank der Arbeit von Rolf Lieberwirth als Vizepräsident vollauf gelungen. Durch sein Wirken fand die Sächsische Akademie der Wissenschaften zu Leipzig zurück in eine von Freiheit und Unabhängigkeit geprägte gesamtdeutsche Wissenschaftslandschaft.

Von seinen zahlreichen und vielseitigen wissenschaftlichen Schriften ${ }^{1}$ ist seine Studie zu Eike von Repgow, den Verfasser des Sachsenspiegels, von besonderem Wert. Sie ist

\footnotetext{
Bibliographie (in Fortsetzungen): LÜCK, H. (Hg.). Rolf Lieberwirth. Rechtshistorische Schriften. Weimar - Köln - Wien: Böhlau, 1997, S. 587-600; LÜCK, H. - SCHILDT, B. (Hg.). Recht-Idee-Geschichte. Beiträge zur Rechts- und Ideengeschichte anläßlich des 80. Geburtstages von Rolf Lieberwirth. Köln Weimar - Wien: Böhlau, 2000, S. 721-723; LÜCK, H. (Hg.). Christian Thomasius (1655-1728). Wegbereiter moderner Rechtskultur und Juristenausbildung. Hildesheim u.a.: Olms, 2006, S. 405-408; LÜCK, H. - SCHILDT, B. - JUNKERMANN, P. (Hg.). Redaktion: HEIL, A.-M. Rolf Lieberwirth. Rechtshistorische Schriften II. Halle an der Saale: Junkermann, Peter, 2016, S. 413-415.
} 
bis heute maßgeblich. ${ }^{2}$ Ähnlich bahnbrechend waren die Akademiepublikationen über das sächsisch-magdeburgische Recht als Quelle osteuropäischer Rechtsordnungen (1986), ${ }^{3}$ über das berühmte Privileg des Erzbischofs Wichmann von Magdeburg von 1188 zum Magdeburger Recht (1990) ${ }^{4}$ und zur Sachsenspiegelglosse (1993). ${ }^{5}$ Die Untersuchung über das sächsisch-magdeburgische Recht als Quelle osteuropäischer Rechtsordnungen markiert den Neubeginn der Erforschung der rechtsgeschichtlichen Gemeinsamkeiten zwischen Deutschland und seinen östlichen Nachbarländern - und zwar in enger Kooperation mit den Wissenschaftlern in diesen Ländern. Aus der Retrospektive betrachtet bildeten diese Studien wertvolle Grundlagen, auf denen zwei erfolgreiche Anträge auf Einrichtung von Akademievorhaben aufbauen konnten. ${ }^{6}$ In jenem Kontext entstanden weitere grundlegende Aufsätze auf dem Gebiet der Sachsenspiegelforschung. Zu nennen wäre hier die rechts- und landesgeschichtlich relevanten Studien zu der Sachsenspiegel-Vorrede „Von der Herren Geburt“ (1991), ${ }^{7}$ zum Zusammenhang von Sachsenspiegel und Landesgeschichte $(1993)^{8}$ sowie zur Wirkungsgeschichte des Sachsenspiegels (1993). ${ }^{9}$ Etwa parallel dazu forschte und publizierte Lieberwirth zur Geschichte und Verbreitung des Magdeburger Stadtrechts. Eher als Nebenprodukt entstand 1986 ein kleines Büchlein mit lateinischen Fachbegriffen und Redewendungen aus Recht und Rechtswissenschaft. Niemand konnte ahnen, dass das Buch 5 Auflagen (2007) erreichen würde, ${ }^{10}$ vor allem auch in erfolgreicher Konkurrenz mit einer Vielzahl ähnlicher Nachschlagewerke. Rolf Lieberwirth war von 1994 bis 2011 Mitglied der Zentraldirektion der Monumenta Germaniae Historica (MGH) und leistete in diesem Amt eine hervorragende Arbeit.

Rolf Lieberwirth gehörte sein ganzes Leben lang zu den aufmerksamen und kritischen Beobachtern der gesellschaftlichen Entwicklung. So hat er auch eine Fülle von Quellen, Eindrücken und Fakten zusammengetragen, welche die jüngere Geschichte der Juristischen Fakultät Halle, an der er über 30 Jahre aktiv gelehrt hat und der er darüber hinaus weitere Jahrzehnte lang verbunden geblieben war, betreffen. Seine Sicht auf die Dinge hat er 2008

2 Eike von Repchow und der Sachsenspiegel (= Sitzungsberichte der Sächsischen Akademie der Wissenschaften zu Leipzig, Philol.-hist. Klasse 122/4). Berlin: Akademie-Verlag, 1982.

3 Das sächsisch-magdeburgische Recht als Quelle osteuropäischer Rechtsordnungen (= Sitzungsberichte der Sächsischen Akademie der Wissenschaften zu Leipzig, Philol.-hist. Klasse 127/1). Berlin: Akademie-Verlag, 1986.

4 Das Privileg des Erzbischofs Wichmann und das Magdeburger Recht (= Sitzungsberichte der Sächsischen Akademie der Wissenschaften zu Leipzig, Philol.-hist. Klasse 130/3). Berlin: Akademie-Verlag, 1990.

$5 \quad$ Über die Glosse zum Sachsenspiegel (= Sitzungsberichte der Sächsischen Akademie der Wissenschaften zu Leipzig, Philol.-hist. Klasse 132/6). Berlin: Akademie-Verlag, 1993.

6 MGH. Sachsenspiegelglossen (Projektleiter: Rolf Lieberwirth); Das sächsisch-magdeburgische Recht als kulturelles Bindeglied zwischen den Rechtsordnungen Ost- und Mitteleuropas (Projektleiter: Heiner Lück).

7 Die Sachsenspiegelvorrede von der herren geburt. In: SCHMIDT-WIEGAND, R. (Hg.). Der Sachsenspiegel als Buch (= Germanistische Arbeiten zu Sprache und Kulturgeschichte 1). Frankfurt am Main u.a.: Lang, 1991, S. 1-18.

8 Entstehung des Sachsenspiegels und Landesgeschichte. In: SCHMIDT-WIEGAND, R. (Hg.). Die Wolfenbütteler Bilderhandschrift des Sachsenspiegels. Aufsätze und Untersuchungen. Kommentarband zur Faksimile-Ausgabe. Berlin: Akademie Verlag, 1993, S. 43-61.

9 Die Wirkungsgeschichte des Sachsenspiegels. In: SCHMIDT-WIEGAND, Die Wolfenbütteler Bilderhandschrift (wie Anm. 8), S. 63-86.

10 Latein im Recht. 5. Aufl. Berlin: Huss-Medien, 2005. 
in einer viel beachteten Monographie veröffentlicht. ${ }^{11}$ Eine solche wissenschaftliche Darstellung der Geschichte einer Juristischen Fakultät (1945-1989) ist in der Bundesrepublik einzigartig. Es gibt wohl keinen weiteren Rechtslehrer, der ein halbes Jahrhundert lang die Entwicklungen vor Ort bewusst registrierte und in eine wissenschaftlich belastbare literarische Form brachte. Das ist Rolf Lieberwirth vollauf gelungen. In diesem Buch kann man gut nachlesen, wie schwer es war, in der DDR über die Grenzen hinaus akzeptierte Wissenschaft zu betreiben und jungen Menschen relativ unabhängig von der verordneten Ideologie fundamentale humanistische und liberale Grundwerte zu vermitteln. Dazu hat Rolf Lieberwirth einen herausragenden Beitrag geleistet.

Während seines wissenschaftlichen Wirkens hat Rolf Lieberwirth nicht nur Fachleuten, sondern auch interessierten Laien durch viele Vorträge und andere Veranstaltungen lohnende Einblicke in seine Forschungen vermittelt.

In Ansehung seiner wissenschaftlichen und wissenschaftsorganisatorischen Leistungen wurde Rolf Lieberwirth verdient mehrfach geehrt. 1990 erschien ihm zu Ehren eine Festschrift anlässlich des 70. Geburtstages. Im Jahr 1995 verlieh ihm die Juristische Fakultät der Georg-August-Universität zu Göttingen die juristischen Ehrendoktorwürde. Zwei Jahre später erschien eine Sammlung seiner Aufsätze. 1998 folgten eine Festschrift zum 75. Geburtstag und 2000 eine weitere Festschrift zum 80. Geburtstag. 2010, anlässlich des 90. Geburtstages, wurde ihm das Bundesverdienstkreuz verliehen. Seine anhaltende wissenschaftlich-schriftstellerische Produktivität veranlasste eine weitere Aufsatzsammlung, die 2016 aus Anlass seines 95. Geburtstages vorgelegt werden konnte.

Bis in sein 99. Lebensjahr hinein war der beeindruckende Gelehrte geistesgegenwärtig, gut über die Tagesereignisse informiert und kommunikativ. Am 5. April 2019 ist Rolf Lieberwirth nach einem langen erfüllten Leben in Halle verstorben. Eine Woche später ist er auf dem Gertraudenfriedhof beerdigt worden. Neben seiner Familie haben ihm der Rektor der Martin-Luther-Universität Halle-Wittenberg, der Dekan der Juristischen und Wirtschaftswissenschaftlichen Fakultät, seine Schüler, Freunde und Weggefährten die letzte Ehre erwiesen.

Wir werden den großen hallischen Rechtshistoriker mit internationaler Strahlkraft, unseren ehemaligen Kollegen und den herzensguten Menschen Rolf Lieberwirth in immerwährender dankbarer Erinnerung behalten.

Heiner Lück

doi: 10.14712/2464689X.2019.38

11 Geschichte der Juristischen Fakultät der Universität Halle-Wittenberg nach 1945. Fakten und Erinnerungen. Köln u. a.: Heymanns, 2008; 2. Aufl. 2010. 